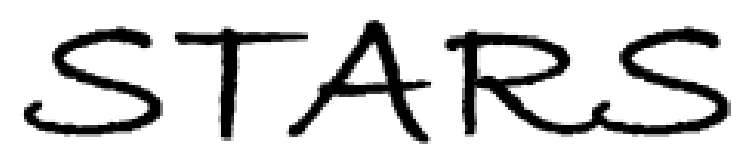

University of Central Florida

STARS

$1-1-2001$

\title{
Morphological operations on color images
}

\author{
Lloyd J. Sartor \\ Arthur R. Weeks \\ University of Central Florida
}

Find similar works at: https://stars.library.ucf.edu/facultybib2000 University of Central Florida Libraries http://library.ucf.edu

This Article is brought to you for free and open access by the Faculty Bibliography at STARS. It has been accepted for inclusion in Faculty Bibliography 2000s by an authorized administrator of STARS. For more information, please contact STARS@ucf.edu.

\section{Recommended Citation}

Sartor, Lloyd J. and Weeks, Arthur R., "Morphological operations on color images" (2001). Faculty Bibliography 2000s. 8200.

https://stars.library.ucf.edu/facultybib2000/8200

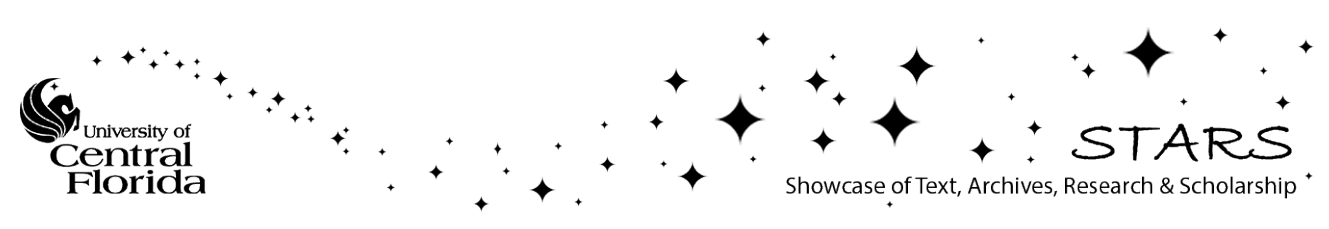




\title{
Morphological operations on color images
}

\author{
Lloyd J. Sartor \\ Siemens Information and Communication Networks \\ 400 Rinehart Road \\ Lake Mary, Florida 32708 \\ Arthur R. Weeks \\ Electrical and Computer Engineering \\ University of Central Florida \\ P.O. Box 25000 \\ Orlando, Florida 32816 \\ E-mail: arw4512@aol.com
}

\begin{abstract}
Morphological image processing has been widely used to process binary and grayscale images, with morphological techniques being applied to noise reduction, image enhancement, and feature detection. Relying on an ordering of the data, morphology modifies the geometrical aspects of an image: object contours in binary images and object surfaces in grayscale images. Extending morphological operators to color image processing has been problematic because it is not easy to define geometry of a vector-valued function and ordering of vectors is not straightforward. We propose a new set of color morphological operators based on a combination of reduced ordering and conditional ordering of the underlying data. (C) 2001 SPIE and IS\&T. [DOI: 10.1117/1.1353199]
\end{abstract}

\section{Introduction}

Morphological image processing is a nonlinear image processing technique with applications in filtering, enhancement, feature extraction, and compression. ${ }^{1}$ The term "morphological" indicates that some aspect of shape in the image is being operated upon. Although based on geometrical concepts, morphological operators can be defined in purely algebraic and set theoretic terms. In a binary image, morphological operations alter an object's contour while in a grayscale image, the shape of the intensity surface is altered. It is easy to visualize the geometry of these image types and to describe the geometrical effects of morphological operators. In a color image, each pixel is vectorvalued, such as a triplet of RGB (red/green/blue), HSI (hue/ saturation/intensity), or $C-Y$ (color difference) values, not scalar-valued as in the binary and grayscale cases. It is difficult to visualize the geometry of these vector-valued functions.

Binary morphology was originally developed as an experimental technique for texture analysis and was later extended to grayscale images. ${ }^{2}$ More recently, the theory has been generalized to complete lattices, of which binary morphology and grayscale morphology are specific instances. ${ }^{3}$

Paper 99036 received June 18, 1999; revised manuscript received Sep. 11, 2000; accepted for publication Sep. 25, 2000

$1017-9909 / 2001 / \$ 15.00$ @ 2001 SPIE and IS\&T
The power of the lattice approach is that morphological operators can be constructed for any set that can be represented by a lattice. (Appendix A provides an introduction to partially ordered sets, totally ordered sets, and lattice theory.) Serra $^{3}$ provides a rigorous mathematical treatment of extending morphological operators to vector valued functions. As stated in his paper, if a property of grayscale morphology depends on the total ordering of luminance, then that property cannot be simply extended to color images. Serra concluded that the color space forms a partially ordered, but not totally ordered, set. For example, grayscale morphological operators can be constructed using threshold set decomposition that relies on the total ordering of the grayscale values. To apply this same approach to color images, one must define a threshold and a threshold set for color images, as well as reconstruction of a color image from its threshold sets. The definition of these concepts for color images is not obvious.

Trahanias and Venetsanopoulos ${ }^{4}$ summarize several techniques for ordering of multivariate data that were originally presented by Barnett. ${ }^{5}$ The most common techniques are marginal ordering ( $M$ ordering), reduced ordering $(R$ ordering), and conditional ordering ( $C$ ordering). Let $\mathbf{X}$ represent a $p$-dimensional vector $\mathbf{X}=\left[X_{1}, X_{2}, \ldots X_{p}\right]^{T}$. The input data consist of $N p$-dimensional vectors, with $\mathbf{X}^{i}$ representing the $i$ th (unordered) sample and $\mathbf{X}^{(i)}$ the $i$ th order statistic. In color image processing applications, each $\mathbf{X}^{i}$ sample is a color vector within the window of the operator. With $M$ ordering, each component is ordered independently. The $i$ th order statistic is $\mathbf{X}^{(i)}$ $=\left[X_{1}^{(i)}, X_{2}^{(i)}, \ldots X_{p}^{(i)}\right]^{T}$. In contrast to scalar order statistics, the marginal order statistic $\mathbf{X}^{(i)}$ may not correspond to any of the original samples.

In $R$ ordering, a scalar measurement is computed for each sample, and then the samples are ordered according to this measurement. A commonly used metric is the generalized distance to a reference point $\alpha$ using a covariance matrix $\Gamma$ that represents the reliability or scale of the measurement in each direction: 
$d_{i}=\left(\mathbf{X}^{i}-\alpha\right)^{T} \Gamma^{-1}\left(\mathbf{X}^{i}-\alpha\right)$.

A problem exists in $R$ ordering when two distinct samples yield the same metric: The ordering of samples with identical metrics is not specified, even though the sample values may be different.

In $C$ ordering, the samples are ordered using one component initially. In the case where multiple samples have the same initial component value, a secondary component is used to order those samples, and so on. $C$ ordering places a total ordering on the data and each order statistic corresponds to an original sample. This approach may make sense where a priority can be placed on the components. But this is not the case when dealing with the RGB color space: each component has equal weighting.

Use of marginal ordering in color image processing algorithms is the most straightforward approach. Several order-statistic filters based on $M$ ordering have been proposed, ${ }^{6}$ including the marginal rank order filter, the marginal median filter, the multichannel $\alpha$-trimmed mean filter, the multichannel modified trimmed mean filter, the multichannel double-window modified trimmed mean filter, and the multivariate $L$ filters. The analysis of these filters using the multivariate probability functions is complex. ${ }^{7}$ Since the output of these filters is not necessarily one of the input samples, objectionable color distortion may occur. Astola et $a l^{8}{ }^{8}$ present the vector median and vector $\alpha$-trimmed mean filters that use reduced ordering of vectors. In the vector median filter, the aggregate distance to all sample vectors is computed for each sample. The sample with the smallest aggregate distance measurement is defined as the vector median because it is the most centrally located vector. The vector $\alpha$-trimmed mean filter computes the same distance measurement for each sample as the vector median. The outlying vectors with the largest measurements are then excluded and the remaining vectors are averaged to produce the filter output. Trahanias and Venetsanopoulos ${ }^{9}$ also presented vector directional filters that separated processing into two stages: directional and magnitude. Directional processing uses an angular measurement function (i.e., reduced ordering) to identify a central group of vectors with approximately the same direction; other vectors are deemed to be outliers and are eliminated. Magnitude processing is then applied to this central group using standard grayscale techniques.

Comer and Delp ${ }^{10}$ present two methods of color morphology: a component-wise method and a vector method. The component-wise method is the same as marginal ordering where each of the RGB components is independently operated upon; i.e., the grayscale algorithm is applied to each RGB component. When using the component-wise method, the output pixel value may not be one of the input pixel values resulting in a new color. This particular method may introduce visually objectionable changes in color and may also be unacceptable in applications that use color for object recognition.

The vector method of Comer and Delp is an application of reduced ordering. A scalar measurement function $d\left(\mathbf{x}_{i}\right)$ is computed for each input color vector $\mathbf{x}_{i}$. The color vectors are ordered according to this measurement. Dilation selects the input color vector with the largest measure $d\left(\mathbf{x}_{i}\right)$ and erosion selects the input color vector with the smallest measure $d\left(\mathbf{x}_{i}\right)$. Opening and closing are defined as the standard iterations of erosion and dilation. The vector method avoids introduction of new colors because the output value is constrained to be one of the input values. If there is no unique largest (or smallest) measure, the authors mention that the output can be chosen based on positioning; no further analysis or example of this condition is made. Specifically, in one example, luminance is used as the ordering function; but the authors do not state how an output is chosen if two different color vectors have the same luminance. The authors discuss two measurement functions: linear combinations of the tristimulus values and the Euclidean norm. The vector method using linear measurement functions is equivalent to a coordinate transformation of the color vectors, where the linear measurement function specifies the value of the primary coordinate; a grayscale morphological operator is then applied to the primary coordinate. Nonlinear measurement functions are more interesting because they can produce vector orderings not possible with simple linear coordinate transformations. Comer and Delp use the Euclidean norm (i.e., the distance to the origin of the coordinate system) as a nonlinear measurement function.

Peters ${ }^{11}$ proposed conditional ordering of color vectors using hue, saturation, and luminance. He uses the $L^{*} u^{*} v^{*}$ color space because distances between color vectors match perceived color differences. The $\left(u^{*}, v^{*}\right)$ coordinates are converted to polar coordinates to represent the perceptually significant attributes of color: The angular coordinate is hue and the radial coordinate is saturation. Luminance and saturation are totally ordered, however hue is not. We cannot say, for example, that green is less than blue. Hue also is a modulo coordinate: a hue angle of $\omega$ is equivalent to a hue angle of $\omega+2 \pi$. Therefore hues close to $2 \pi$ are similar to hues close to zero, although there is a large numerical difference in their values. Additionally, the selection of which color corresponds to the zero reference hue is arbitrary. A hue angle of zero can be red, blue, green, etc. To order hues, Peters uses a hue-valued structuring function. Hues are ordered according to the absolute value of the difference between the image hue value and the corresponding structuring function hue value. Since hue is modulo $2 \pi$, the absolute hue difference is normalized to a value within $[0$, $\pi]$. Dilation of hue is defined as selection of the image hue value that generates the largest absolute hue difference measurement. Likewise, erosion of hue is defined as selection of the image hue value that generates the smallest absolute hue difference measurement. Assuming a constant structuring element, the erosion operator biases the output toward the structuring element hue, and dilation to the opposite (complementary) hue. Since the output vector always corresponds to one of the input vectors, no color artifacts are introduced. Peters defines a set of color morphological operators using conditional ordering of absolute hue difference, saturation, and luminance. This method has a shortcoming in that the use of absolute value of hue difference for ordering of hue does not form a lattice, and consequently ambiguity in proper selection of the operator's output may result. This is evident when two pixels within the window have identical absolute hue difference, saturation, and luminance, but whose hues are different. This can arise with a constant structuring element and the image hues are 
on opposite sides of the structuring element hue, or it can happen when the structuring element hues are allowed to vary. The method does not specify how the infimum or supremum is chosen in these cases. A complete lattice requires that every pair of lattice elements have unique infimum and supremum.

In order to define color morphological operators consistent with the lattice framework, the set of all possible color images must form a complete lattice. However, in order to define the lattice of color images and color morphological operators, the color vectors must be ordered in some fashion. It is not clear if this must be a total ordering as in the grayscale case, or a partial ordering such that the set of possible color vectors forms a complete lattice. The ordering of color vectors is the fundamental problem in defining a color image morphology.

We propose a color vector ordering technique that allows color morphological operators to be defined. Section 2 provides a brief review of binary and grayscale operators. In Sec. 3, color coordinate systems are discussed and the proposed color morphological operators are defined. Section 4 presents results obtained using these color morphological operators. Section 5 summarizes our conclusions. The Appendix provides a brief introduction to lattice theory and showing that the proposed color morphological operators fit within the lattice framework.

\section{Binary and Grayscale Morphological Operators}

Binary dilation and erosion are defined using Minkowski set addition and Minkowski set subtraction, respectively:

$X \oplus B=\underset{b \in B}{\cup} X_{b}$

and

$X \ominus B=\cap X_{-b}$,

where $X$ is the binary image, $B$ is a set called the structuring element, and $X_{b}$ is the translate of $X$ by $b$. (The translate of set $X$ by point $b$ is $\{x+b \mid x \in X\}$.) Minkowski addition is the union of the image translates to each point within the structuring element. Minkowski subtraction is the intersection of the image translates to each point in the reflected structuring element (i.e., rotated $180^{\circ}$ about the origin). The structuring element can be considered as part of the erosion and dilation operators, as the operator and structuring element together determine the geometry of the filtered object. Binary dilation can be described simply as sliding the center of the structuring element on the perimeter of the object. The dilated object is the original object augmented by the additional area covered by the structuring element as it moves around the perimeter. If the structuring element is circular, then dilation can be pictured as rolling a circle on the perimeter of the object, and any area covered by the circle becomes part of the dilated object. With a circular structuring element, binary erosion can be pictured as rolling the circle on the inside perimeter of the object, and any traversed area is removed from the original object to form the eroded object.
Grayscale dilation and erosion are defined as

$$
[f \oplus g](x)=\max _{\substack{x \in D \\ z-x \in G}}\{f(z)+g(z-x)\}
$$

and

$[f \ominus g](x)=\min _{\substack{z \in D \\ z-x \in G}}\{f(z)-g(z-x)\}$,

where $g(x)$ is the structuring function. The domain (also called the support) of the structuring function determines the shape and size of the sliding window of the morphological operators. The values of the structuring function affect the shape of the resulting intensity surface. If $g(x)$ $=0$ within its support, then the dilation and erosion operators are called flat operators and reduce to

$$
\begin{gathered}
{[f \oplus g](x)=\max _{\substack{z \in D \\
z-x \in G}}\{f(z)\},} \\
{[f \ominus g](x)=\min _{\substack{z \in D \\
z+x \in G}}\{f(z)\},}
\end{gathered}
$$

and are equivalent to the $n$ th-ranked order filter (max filter) and first-ranked order filter (min filter), respectively. Grayscale dilation can be interpreted as rolling a sphere on the surface of the function; the points transcribed by the center of the sphere define the dilated image. In the same fashion, grayscale erosion can be interpreted by rolling a sphere on the bottom of the image's surface. It is easy to see that dilation enhances bright areas while diminishing dark areas, and vice versa for erosion.

Two other fundamental morphological operators, opening and closing, are defined in terms of dilation and erosion (for both the binary and grayscale case). Opening is erosion followed by dilation and closing is dilation followed by erosion:

$r \circ s=(r \ominus s) \oplus s$,
$r \cdot s=(r \oplus s) \ominus s$.

Opening smooths extrusions on binary contours and grayscale surfaces, whereas closing smooths indentations.

\section{Color Morphological Operators}

Several coordinate systems are available for representing color images. The most common is the RGB system that specifies the intensities of the red, green, and blue primary colors. The RGB system is used for most image capture and display devices, including desktop computer monitors. Although convenient for electronics, the RGB system is often not appropriate for image processing applications because it does not represent the perceptual attributes of hue, saturation, and intensity, where the hue identifies the dominant wavelength, the saturation represents the amount of white light mixed with the pure color, and the intensity indicates the color's brightness. The components of the HSI color model are the perceptual attributes of color: hue, saturation, and intensity. Disadvantages of using the HSI space 
in image processing applications are the complex conversion from RGB space and the lack of gamma correction for the perceived brightness of the red, green, and blue primaries. Color television uses the color difference $(C-Y)$ color system, which separates chromatic information from luminance information. A single coordinate $(Y)$ represents the luminance information and the other two coordinates $(R-Y$ and $B-Y)$ represent the chromatic information. The chromatic components can be converted to polar coordinates to represent hue and saturation.

Any other trichromatic color space can be used to represent the color components of an image, such as the YIQ, HLS, $L^{*} U^{*} V^{*}, L^{*} a^{*} b$, or any of the CIE color spaces. ${ }^{1}$ We chose to use the $C-Y$ color space because it can model the perceptually significant attributes of color and because the RGB to $C-Y$ transformation and its inverse are simple to implement and use only linear transformations. Additionally, the $C-Y$ color space was chosen because it is one of the standards used for color television, making hardware implementation of the proposed color morphological operations more feasible. Furthermore, the luminance $(Y)$ component of the $C-Y$ color model is gamma corrected in accordance with the perceived brightness for the red, green, and blue components, and is not merely their average value. $^{12}$

Figure 1(a) shows the RGB color space used to represent 24-bit color images. Each of the red, green, and blue color components have 256 distinct intensities, allowing for 16.7 million possible colors. Located at six of the eight corners of the RGB cube are the six primary and secondary colors. The line connecting the other two corners (black to white) represents the possible grayscale intensities available in a black and white image.

Figure 1(b) illustrates the chromatic plane including the location of the red, green, and blue primary colors. This is the projection of the realizable colors of Fig. 1(a) onto the $R-Y / B-Y$ plane. The equations describing the RGB to $C-Y$ transformation are ${ }^{1}$

$$
\left[\begin{array}{c}
Y \\
(R-Y) \\
(B-Y)
\end{array}\right]=\left[\begin{array}{ccc}
0.299 & 0.587 & 0.114 \\
0.701 & -0.587 & -0.114 \\
-0.299 & -0.587 & 0.886
\end{array}\right]\left[\begin{array}{c}
R \\
G \\
B
\end{array}\right]
$$

where $R, G$, and $B$ are the red, green, and blue components of the original RGB image. The third color difference ( $G$ $-Y)$ is typically written in terms of $(R-Y)$ and $(B-Y)$ as

$(G-Y)=-0.51 \cdot(R-Y)-0.19 \cdot(B-Y)$.

The inverse transformation from $C-Y$ to RGB computes the red, green, and blue intensities by adding the luminance value $Y$ back to the $(R-Y),(B-Y)$, and $(G$ $-Y$ ) components. The $C-Y$ coordinates are often converted into polar coordinates to represent the perceptual attributes of hue and saturation as indicated in Fig. 1(c). These values are computed using

$$
H=\tan ^{-1}\left[\frac{(B-Y)}{(R-Y)}\right],
$$

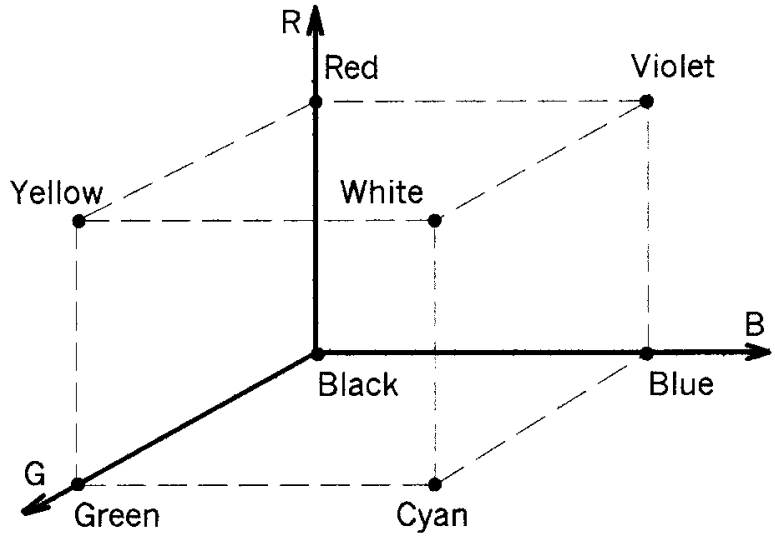

(a)

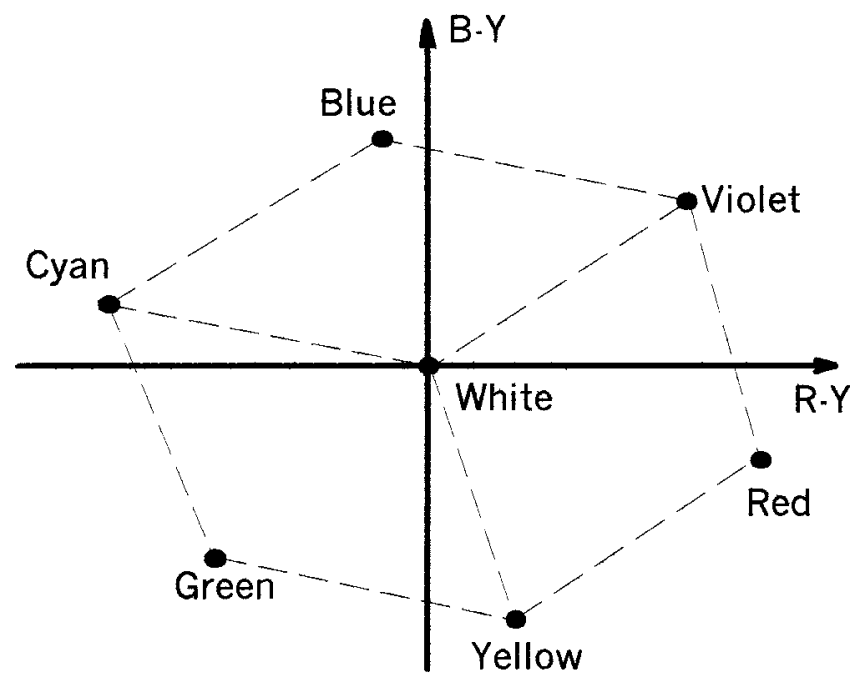

(b)

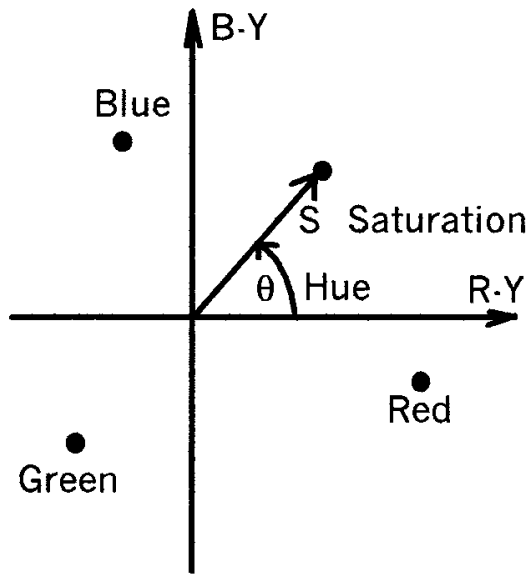

(c)

Fig. 1 (a) The RGB color space, (b) a plot of the $C-Y$ color space showing the orientation of the primary and secondary colors, and (c) the definition of the hue and saturation color components in the $C-Y$ color space.

$S=\sqrt{(R-Y)^{2}+(B-Y)^{2}}$,

where the saturation $S$ is the length of the vector from the origin of the chromatic plane to the specific color, and the hue $H$ is the angle between the $(R-Y)$ axis and the satu- 
ration vector. Grayscale images have a saturation value of zero, hence, all grayscale intensities map to the origin of the $C-Y$ chromatic color plane (i.e., $B-Y=R-Y=0$ ) and have an undefined hue value.

Just as we can say "binary dilation makes objects larger" and '"grayscale dilation makes objects brighter," we would like to make general qualitative statements about color morphological operations. In grayscale morphology, the dilation operation shifts the image toward the maximum of the grayscale. In a perceptual color space, there is no singular corresponding maximum value nor minimum value. The luminance and saturation components are totally ordered, so therefore have maximum and minimum values. However, the hue component is a modulo $2 \pi$, angular component and does not have maximum nor minimum values.

For the proposed color morphological operators, we define a reference color that is analogous to the maximum graylevel in grayscale morphology: Color dilation will tend to move toward this reference color and color erosion away from it. We can then speak of red dilation, blue erosion, or green opening. A red dilation should increase the size and brightness of red objects within the image; a blue erosion should decrease the size and brightness of blue objects; and a green opening should smooth irregularities in green objects. It will be shown that the reference color must have maximum luminance and maximum saturation to allow ordering of color vectors. The hue of the reference color used for a particular morphological operation is determined by the application.

Color vectors within the structuring element support are ordered according to their generalized distance to the reference color:

$d_{i}=\left(\mathbf{X}^{i}-\alpha\right)^{T} \Gamma^{-1}\left(\mathbf{X}^{i}-\alpha\right)$,

where $\alpha$ is the reference color vector, $\mathbf{X}^{i}$ is a color vector within the operator's window, and $\Gamma$ is a covariance matrix that represents the reliability or scale of the measurement in each direction. Ordering color vectors using the generalized distance measurement is an application of reduced ordering. Each color vector is composed of a single luminance component $(Y)$ and two orthogonal chromatic components $(R-Y$ and $B-Y)$. The three color components can be treated as orthogonal, yielding $\Gamma^{-1}$ which is diagonal:

$\Gamma^{-1}=\left[\begin{array}{ccc}\gamma_{1} & 0 & 0 \\ 0 & \gamma_{1} & 0 \\ 0 & 0 & \gamma_{3}\end{array}\right]$.

In Fig. 2, a red reference color and a color vector $\mathbf{X}$ within the realizable color space are shown and the corresponding distance $d$ between them. Color dilation and erosion are defined as follows:

$[f \oplus B](x)=\mathbf{X}^{i}: d_{i}<d_{j} \forall \mathbf{X}^{i}, \mathbf{X}^{j} \in f\left(B_{x}\right)$,

$[f \ominus B](x)=\mathbf{X}^{i}: d_{i}>d_{j} \forall \mathbf{X}^{j}, \mathbf{X}^{j} \in f\left(B_{x}\right)$,

where $f$ is the color image, $B$ is the structuring element (that defines the operator's window), $\mathbf{X}^{i}$ and $\mathbf{X}^{j}$ are color vectors within the operator's window, $f\left(B_{x}\right)$ is the set of color

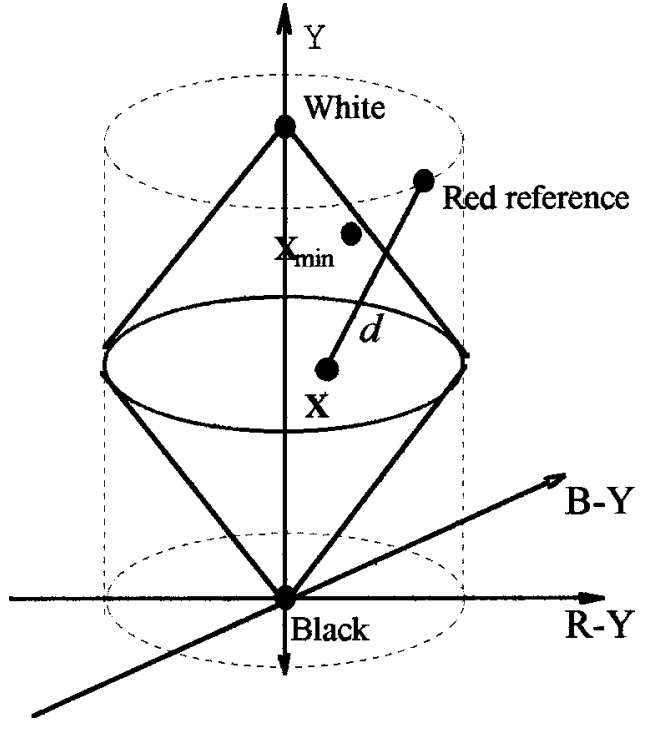

Fig. 2 Three-dimensional plot of the $C-Y$ color space showing the location of red reference color.

vectors in the image that are inside the operator's window, and $d_{j}$ is the distance from $\mathbf{X}^{j}$ to the reference color. Color dilation selects the color vector with minimum distance (i.e., closest to the reference color) and color erosion selects the color vector with maximum distance. This definition causes, for example, a dilation using a red reference color to make the image more red. Color artifacts are avoided because the output color vector always corresponds to one of the input color vectors. (Color opening and closing are defined identically as in the grayscale case: Opening is erosion followed by dilation and closing is dilation followed by erosion.)

In the proposed color dilation and erosion operators, the structuring element is a binary set. This is similar to function- and set-processing (FSP) operators for grayscale morphology as presented by Maragos, ${ }^{13}$ where a binary structuring element is used for grayscale morphological operators. FSP is a special case of function processing where grayscale structuring elements are used for grayscale morphological operators.

Let the reference color and an arbitrary color vector be represented, respectively, by the $C-Y$ coordinates $\left(y_{\max }, r y_{\text {ref }}, b y_{\text {ref }}\right)$ and $(y, r y, b y)$, where the maximum allowable luminance is chosen as the luminance for our reference color. $r y_{\text {ref }}$ and $b y_{\text {ref }}$ are also selected to yield the maximum allowable saturation. The distance measurement can be expressed by:

$d=\gamma_{1}\left(y-y_{\max }\right)^{2}+\gamma_{2}\left(r y-r y_{\mathrm{ref}}\right)^{2}+\gamma_{3}\left(b y-b y_{\mathrm{ref}}\right)^{2}$.

It is possible that two or more distinct color vectors within the operator's window are equidistant from the reference color. To avoid color artifacts, we have constrained the operator to select a color vector from the input samples. Therefore, a means is required to order a set of color vectors equidistant from the reference color.

Color vectors that are equidistant from the reference color lie on a sphere centered at the reference color. Because the human visual system is more sensitive to changes 
in hue than to changes in luminance and saturation, ${ }^{14}$ the first tie-breaking decision selects the color vector with minimum hue, where the reference color is assigned a hue of zero. [Graylevels have an undefined hue according to Eq. (12), so are assigned a hue value of zero.] It is possible that hues near $2 \pi$ are actually closer to the reference color than hues near zero, so use of absolute value may seem appropriate. We do not use absolute value of hue difference because of the ambiguity that may be introduced in hue ordering. Consequently, we have imposed a total ordering on the hue component.

It is possible that two or more distinct color vectors have the same distance metric and the same hue. We chose the next ordering decision to be selection of the color vector with the smallest saturation. Therefore, when multiple color vectors share the minimum hue value and the minimum hue value is significantly different from the reference hue, this ordering decision gives preference to low-saturated colors over high-saturated colors to minimize color artifacts. When the minimum hue value is similar to the reference hue, this ordering decision gives preference to lowersaturated, higher-intensity color vectors over highersaturated, lower-intensity color vectors. If multiple input color vectors have identical distance metric, hue, and saturation, then the color vector with higher luminance is chosen.

Ordering of color vectors is therefore a hybrid of reduced ordering and conditional ordering: The distance measurement for reduced ordering provides the primary ordering and perceptual attributes are used for subsequent ordering when necessary. The complete ordering relation is the conditional ordering of $(d, \omega, s, y)$ :

$$
X_{1}<X_{2} \text { if }\left\{\begin{array}{l}
d_{1}<d_{2} \text { or } \\
d_{1}=d_{2} \text { and }\left\{\begin{array}{l}
\omega_{1}<\omega_{2} \text { or } \\
\omega_{1}=\omega_{2} \text { and }\left\{\begin{array}{l}
s_{1}<s_{2} \text { or } \\
s_{1}=s_{2} \text { and } y_{1} \geqslant y_{2}
\end{array}\right.
\end{array}\right.
\end{array}\right.
$$

( $d$ is the distance measurement, $\omega$ is the hue where the reference color hue is zero, $s$ is saturation, and $y$ is luminance.) If $X_{1}<X_{2}$, the supremum operator selects $X_{2}$ and the infimum operator selects $X_{1}: X_{1} \vee X_{2}=X_{2}$ and $X_{1} \wedge X_{2}$ $=X_{1}$. The color dilation operator is $C$ ordering of $(\min d, \min \omega, \min s, \max y)$. Similarly, the color erosion operator is $C$ ordering of $(\max d, \max \omega, \max s, \min y)$.

Our proposal differs from Peters ${ }^{11}$ in the components used for the conditional ordering. Peters uses only the attributes of hue, saturation, and luminance, whereas we order primarily on a distance measurement and use the perceptual attributes as secondary ordering criteria. Our proposed ordering also totally orders the color space, which avoids ambiguity in selection of an output. Peters does not address how an output value is chosen if two distinct color vectors have identical measurements. To avoid ambiguity, we have fixed the color reference and restricted the structuring element to a binary pattern.

Color morphological operators must also fit within the lattice theory framework for general morphology. The Appendix provides a brief introduction to lattice theory and how binary and grayscale morphology fit within the lattice framework. It then shows how the proposed color morphology also fits within the lattice framework.

Applying a color morphological operator to a grayscale image should be equivalent to applying the corresponding grayscale operator to the image's luminance component. Consider a color image in which saturation is zero for all color vectors: all color vectors lie on the luminance axis and the image is equivalent to a grayscale image. Since these color vectors lie on the luminance axis, they have $C-Y$ coordinates $(y, 0,0)$; i.e., $R-Y=B-Y=0$. Recalling that the reference color is assigned maximum luminance, if the reference color has $C-Y$ coordinates of $\left(y_{\max }, r y_{\text {ref }}, b y_{\text {ref }}\right)$ and the covariance matrix is diagonal, the distance measurement to a color on the luminance axis is

$d=\gamma_{1}\left(y-y_{\text {max }}\right)^{2}+\gamma_{2} r y_{\text {ref }}^{2}+\gamma_{3} b y_{\text {ref }}^{2}$

Since $r y_{\text {ref }}, b y_{\text {ref }}, y_{\text {max }}$, and the $\gamma$ s are constant for all measurements, the distance measure becomes a monotonically decreasing function of $y$; i.e., the distance from the reference color to a point on the luminance axis decreases as luminance increases. The color dilation operator will choose the color vector closest to the reference color; i.e., the one with the highest luminance. Likewise, the color erosion operator will choose the color vector with the lowest luminance. Therefore, color dilation and erosion reduce to grayscale dilation and erosion when applied to grayscale images. This is apparent in Fig. 2: distance to the red reference color increases as the luminance axis is traversed from white to black.

\section{Results}

The color dilation and erosion algorithms were implemented on a desktop computer using the $\mathrm{C}++$ programming language. The RGB color images are converted to $C-Y$ and then to luminance, saturation, and hue using Eqs. (10), (12), and (13). The distance measurement for the primary ordering was implemented with Eq. (14), allowing for an arbitrary covariance matrix $\Gamma$. For the dilation operator, the algorithm selects a color vector within the structuring element support according to the following conditions. (For the erosion operator, the inequalities are reversed.) Note that this is conditional ordering: once a unique color vector is determined, the decision making is complete. 


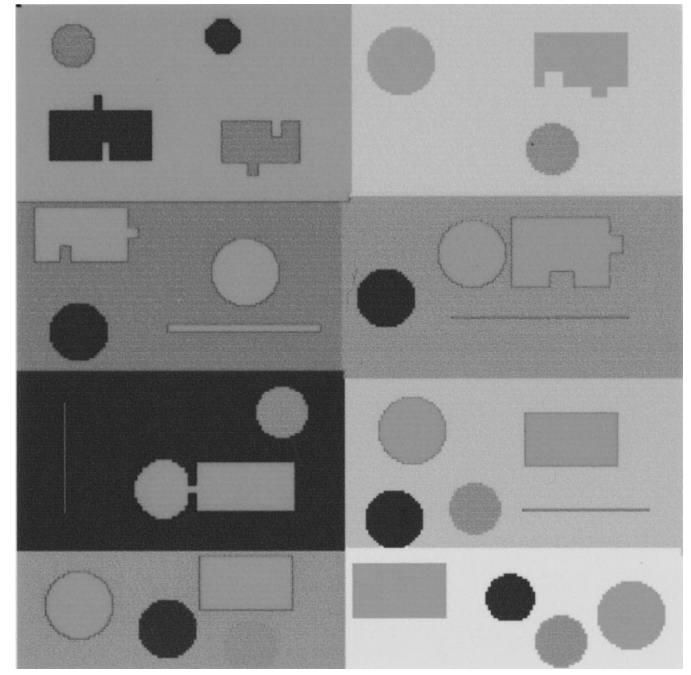

Fig. 3 Synthetic test image.

1. Select the color vector with minimum $d$; if a tie go to 2.

2. Select the color vector with minimum ( $\omega$ $\left.-\omega_{\text {ref }}\right) \bmod 2 \pi$, where $\omega_{\text {ref }}$ is the hue of the reference color, computed using Eq. (12); if a tie go to 3 .

3. Select the color vector with minimum saturation, computed using Eq. (13); if a tie go to 4 .

4. Select the color vector with maximum luminance.

The color morphological operations of dilation, erosion, closing, and opening were performed on a synthetic test image using a $5 \times 5$ pseudocircular structuring element. The synthetic test image consists of simple color geometrical shapes on different color backgrounds, as shown in Fig. 3 . The following covariance matrix was used, which gives equal weight to the $Y, R-Y$, and $B-Y$ components:

$\Gamma^{-1}=\left[\begin{array}{lll}1 & 0 & 0 \\ 0 & 1 & 0 \\ 0 & 0 & 1\end{array}\right]$.

This yields the Euclidean distance from the reference color

$d=\left(y-y_{\max }\right)^{2}+\left(r y-r y_{\text {ref }}\right)^{2}+\left(b y-b y_{\text {ref }}\right)^{2}$.

The morphological operators were applied to the test image using two different reference color vectors: a red reference with a hue of $336^{\circ}$ and a blue reference with a hue of $98^{\circ}$. Figures $4-7$ show the results with the red reference color and Figs. 8-11 show the results with the blue reference color. In the red dilation (Fig. 4), the red objects became larger. Notice in the upper left section how the green and blue extrusions were removed and the red indentations into the green and blue rectangles were enlarged. Red objects that are close together were joined, such as the red circle and red rectangle in the center right. These results are consistent with the qualitative description of red dilation: A red dilation makes red objects larger. An interesting anomaly is the pink background with red shapes in the

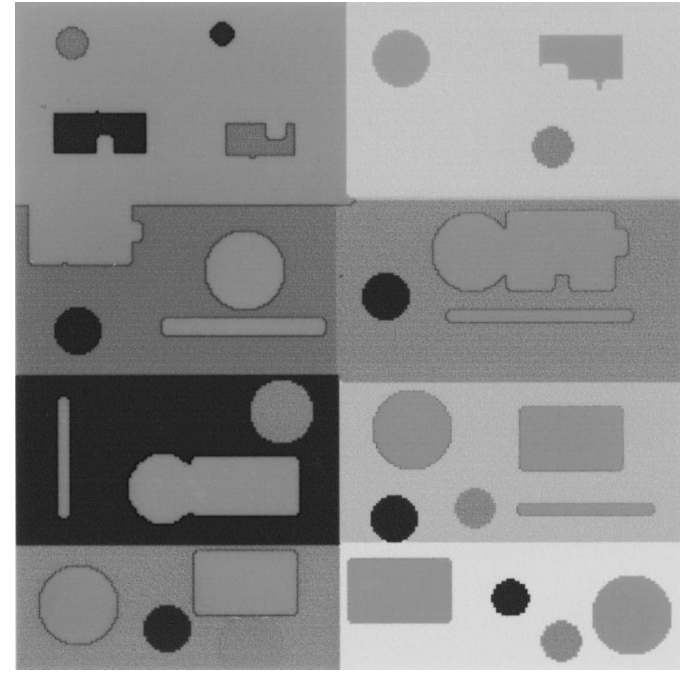

Fig. 4 Red dilation of test image using equal component weights.

upper right. Here the pink background expanded into the red objects, even though the pink is less saturated. The reason is that pink (being a combination of red, green, and blue) has a higher luminance than the bright red background, so therefore is closer to the red reference color. Nonred objects on nonred backgrounds also changed size, such as the green and blue circles throughout the image. Even in the absence of any pixel values that could be considered "red," the pixel value closest to the red reference color is selected as the output. For example, in the lower right, the yellow background (being a mixture of red and green) is closer to the reference color than the blue or green circles, so the circles became smaller.

In the red erosion result in Fig. 5, notice that the narrow red lines have been removed. The small connection between the red circle and red rectangle in the center left has been broken. In the upper left, the small red cutouts in the green and blue objects have been filled. Similar results can be seen in the red closing and red opening of the test image

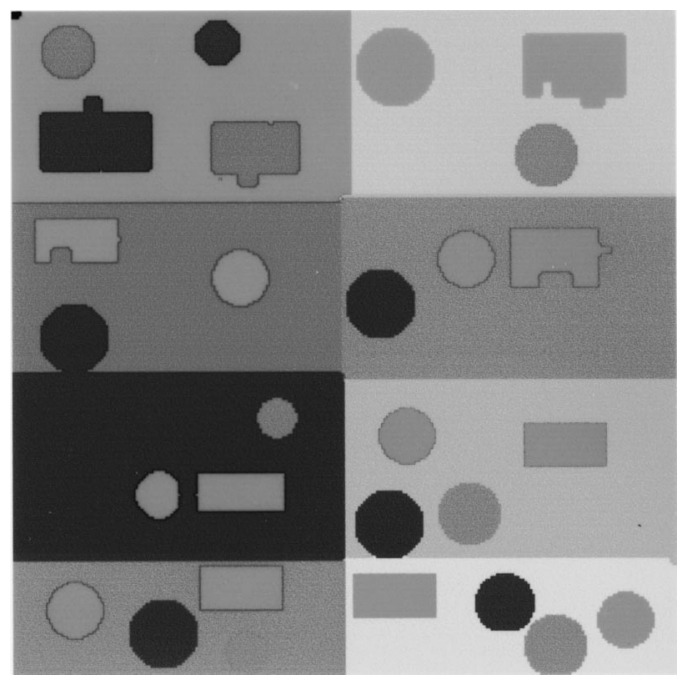

Fig. 5 Red erosion of test image using equal component weights. 


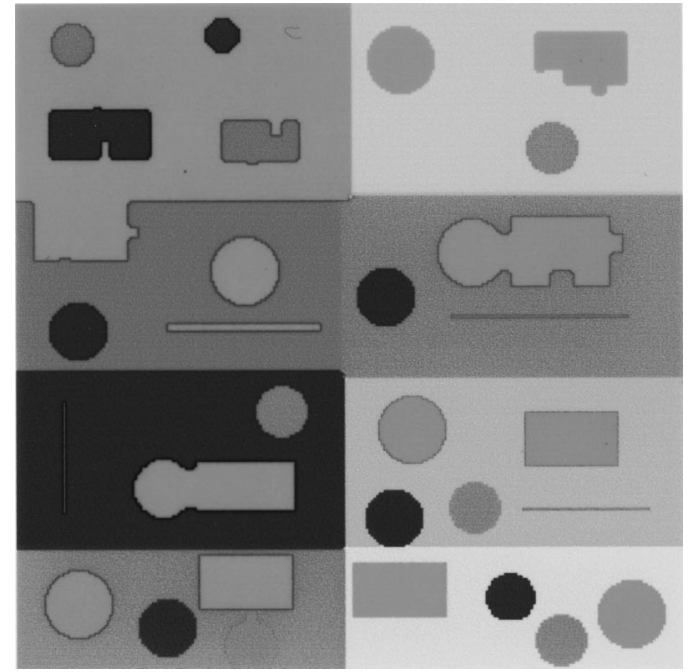

Fig. 6 Red closing of test image using equal component weights.

(Figs. 6 and 7). The rounding effect of the pseudocircular structuring element is apparent in the closed and opened images.

Figure 8 shows the blue dilation of the test image. The blue objects were enlarged-notice that the blue circle and blue region on the left grew into each other so that they touch. The isolated thin red line and the thin red line connecting the red circle and rectangle (on the left) were removed, showing similar results as the red erosion in this region. The bright blue circle in the light blue region at the bottom right actually decreased in size because the light blue has higher luminance than bright blue. Therefore, the light blue color is closer to the reference than the bright blue. Figure 9 shows the blue erosion, that appears similar to the red dilation in many areas. The bright blue areas shrank in size. The blue closing and blue opening are shown in Figs. 10 and 11.

The red morphological operations were repeated using the following covariance matrix that weights the luminance component less heavily than the $R-Y$ and $B-Y$ compo-

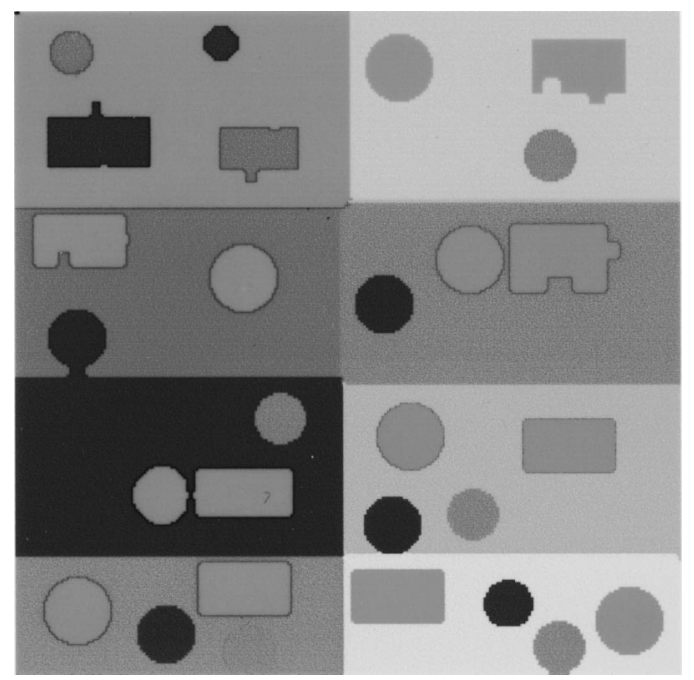

Fig. 7 Red opening of test image using equal component weights.

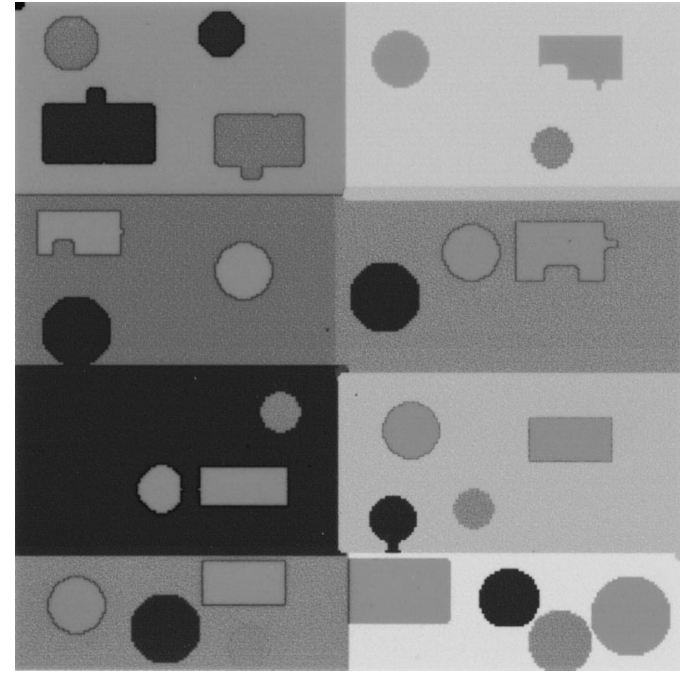

Fig. 8 Blue dilation of test image using equal component weights.

nents. That is, the luminance axis is effectively compressed, decreasing distances in the luminance direction

$\Gamma^{-1}=\left[\begin{array}{ccc}0.1 & 0 & 0 \\ 0 & 1 & 0 \\ 0 & 0 & 1\end{array}\right]$.

The distance measurement then becomes

$d=0.1\left(y-y_{\max }\right)^{2}+\left(r y-r y_{\mathrm{ref}}\right)^{2}+\left(b y-b y_{\mathrm{ref}}\right)^{2}$.

The resulting images are shown in Figs. 12-15. The primary difference between these results and those using equal component weights is in the upper right section where red objects lie on a pink background. With the luminancereduced weighting, the red color vectors are closer to the reference color than the pink color vectors. In the dilation (Fig. 12), the red objects increased in size, as one would intuitively expect. In the erosion (Fig. 13), the red objects

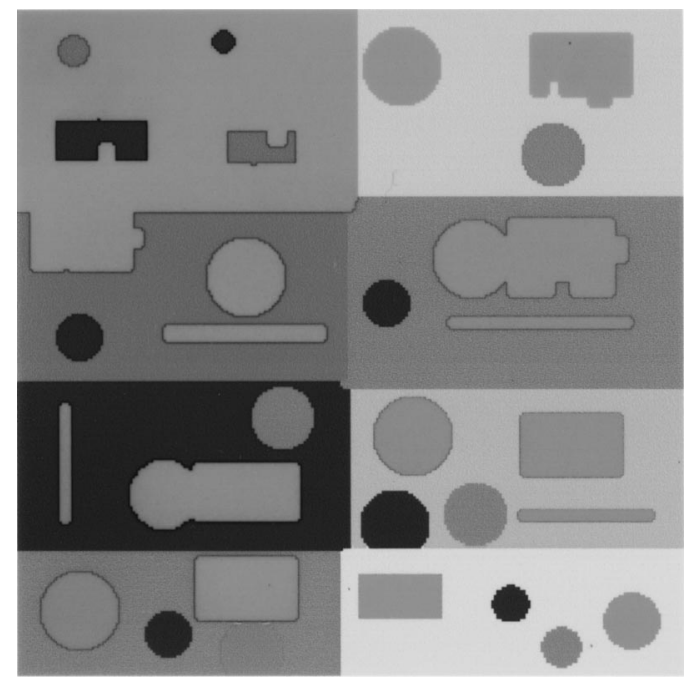

Fig. 9 Blue erosion of test image using equal component weights. 


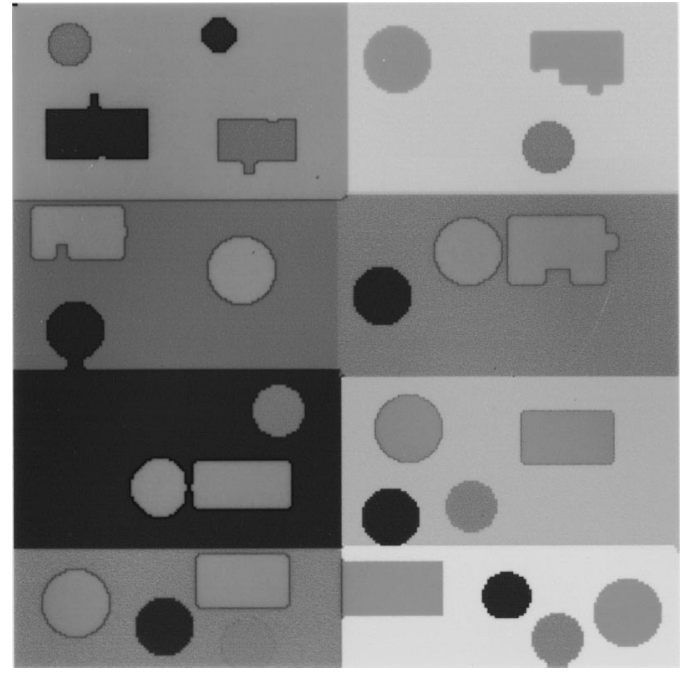

Fig. 10 Blue closing of test image using equal component weights.

decrease in size. The only other difference is in the lower left section where the green circle on the cyan background decreased in size when using the luminance-reduced weighting, whereas it increased in size with the equallyweighted covariance matrix.

\section{Conclusion}

Color morphological operations are based on ordering of color vectors. It is preferable to represent the color vectors in a perceptual space, such as the $C-Y$ color difference space with the $R-Y / B-Y$ components converted to polar coordinates representing the perceptual attributes of hue and saturation. We propose a color vector ordering as a hybrid of reduced ordering and conditional ordering. The generalized distance to a reference color vector provides the primary ordering criterion. The reference color, which specifies the supremum of the color vector ordering, is parametrized by hue. The covariance matrix also plays a significant role in ordering. We found that weighting the luminance component less heavily than the chromatic

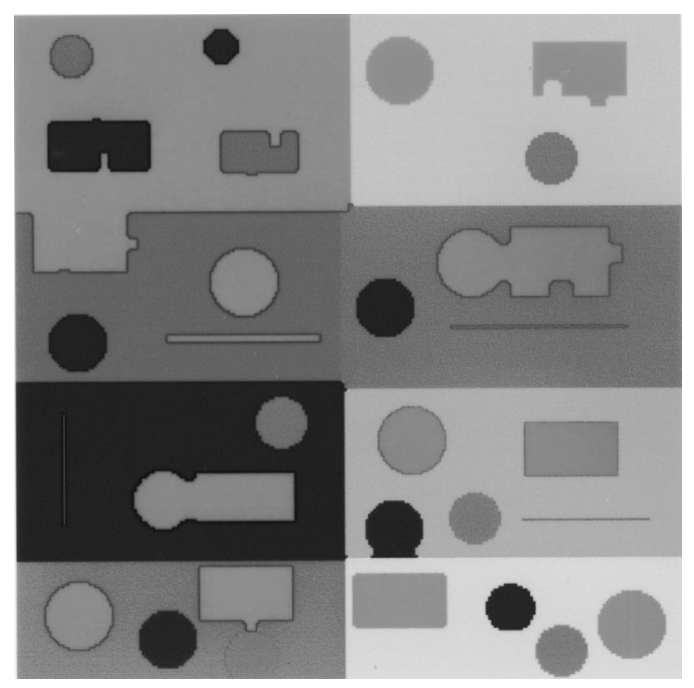

Fig. 11 Blue opening of test image using equal component weights.

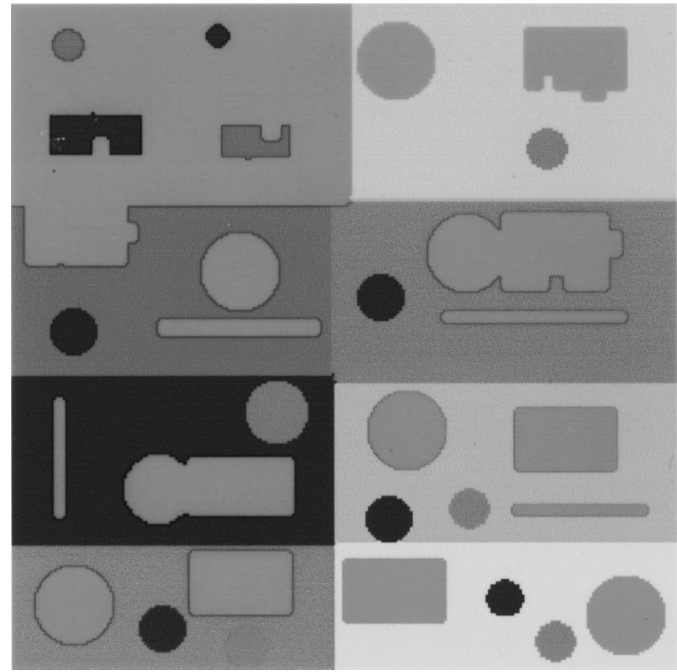

Fig. 12 Red dilation of test image with luminance weighted less than the $R-Y$ and $B-Y$ components $\left(\gamma_{1}=0.1\right)$.

components produced better results for the case when objects are the same hue but differ in saturation and luminance. The resulting total ordering of color vectors allows color morphology to fit within the complete lattice framework.

\section{Appendix: Introduction to Lattice Theory ${ }^{15}$}

A partially ordered set (poset) is a set in which a partialordering relationship exists between its members. This partial-ordering relationship is denoted by the symbol $<$ and has the following properties, where $X$ and $Y$ denote members of the set:

(a) $X<X \quad$ (reflexivity);

(b) $X<Y$ and $Y<X$ implies $X=Y \quad$ (antisymmetry);

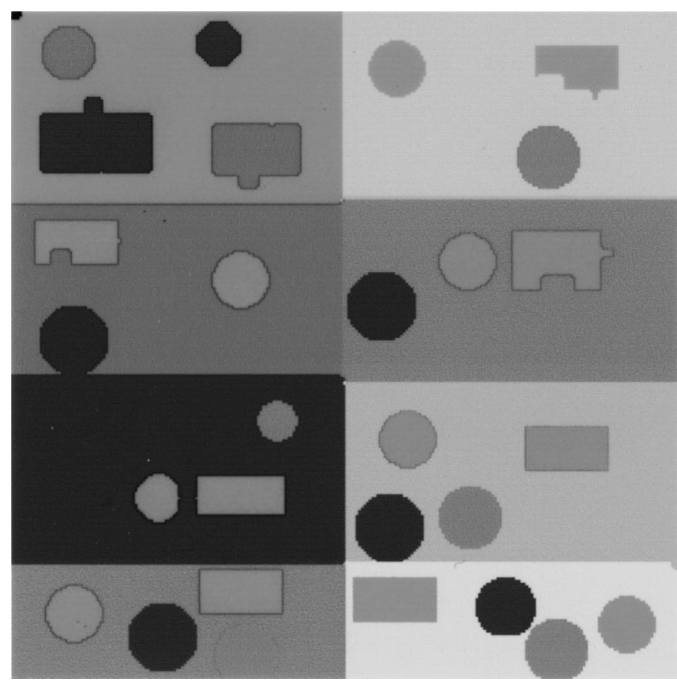

Fig. 13 Red erosion of test image with luminance weighted less than the $R-Y$ and $B-Y$ components $\left(\gamma_{1}=0.1\right)$. 


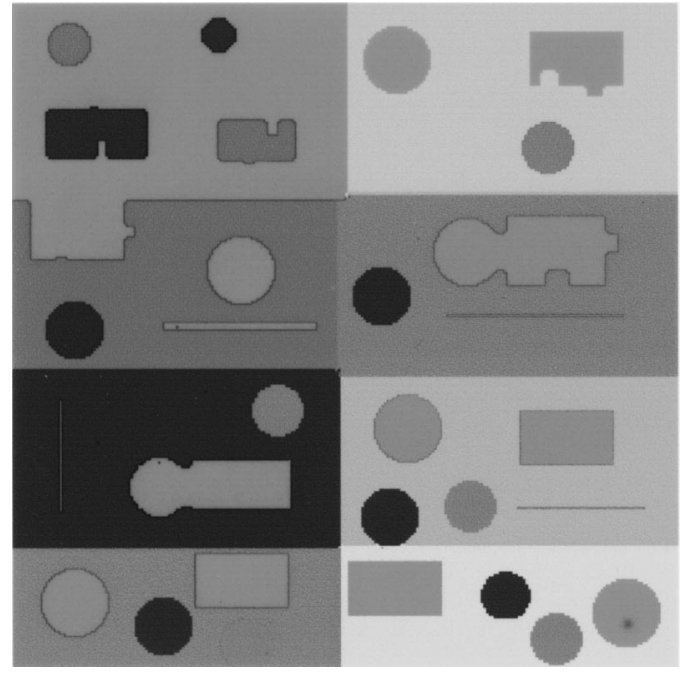

Fig. 14 Red closing of test image with luminance weighted less than the $R-Y$ and $B-Y$ components $\left(\gamma_{1}=0.1\right)$.

(c) $X<Y$ and $Y<Z$ implies $X<Z$ (transitivity).

(Sometimes the symbol $\leqslant$ is used for the partial-ordering relation. It is important to realize that the symbol represents an ordering relation and not inequality.) A set is totally ordered if the following property (which we call pairwise ordering) also holds:

(d) $X<Y$ or $Y<X$

for every pair $X, Y \in \mathcal{L}$ (pairwise ordering).

$A$ is a lower bound of a poset $\mathcal{L}$ if $A<X$ for all $X \in \mathcal{L}$. Likewise, $B$ is an upper bound of a poset $\mathcal{L}$ if $X<B$ for all $X \in \mathcal{L}$. (Note that $A$ and $B$ are not necessarily members of $\mathcal{L}$.) Let $A^{\prime}$ be the set of all lower bounds of $\mathcal{L} ; A$ is the greatest lower bound (g.l.b.) of $\mathcal{L}$ if $A_{0}<A$ for all $A_{0}$

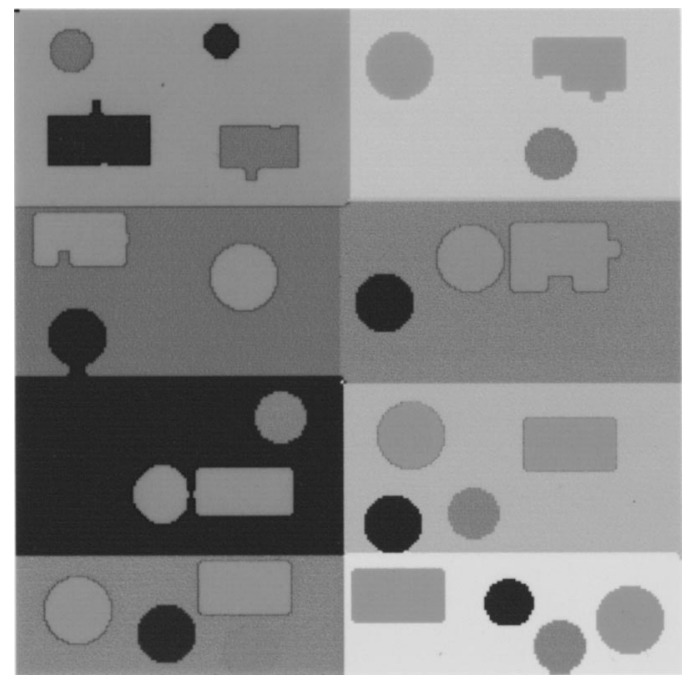

Fig. 15 Red opening of test image with luminance weighted less than the $R-Y$ and $B-Y$ components $\left(\gamma_{1}=0.1\right)$. $\in A^{\prime}$. Similarly, $B$ is the least upper bound (1.u.b.) of $\mathcal{L}$ if $B<B_{0}$ for all $B_{0} \in B^{\prime}$, where $B^{\prime}$ is the set of all upper bounds of $\mathcal{L}$.

A lattice is a poset in which any two elements possess a 1.u.b. and a g.l.b. We say that lattice element $A$ is "below" $B$ if $A<B$. The complementary relation is $\nless: A \nless B$ means $A$ is not below $B$. Note that $A \nless B$ does not imply $B<A$. The ordering relation does not necessarily apply to every pair of lattice members; i.e., there may exists elements $A$ and $B$ such that $A \nless B$ and $B \nless A$. Every pair of lattice elements $A$ and $B$ do have an l.u.b. called the supremum of $A$ and $B$; both $A$ and $B$ are below the supremum. Also, $A$ and $B$ have a g.l.b. called the infimum of $A$ and $B$; the infimum is below both $A$ and $B$. The supremum and infimum operators are represented by the symbols $\vee$ and $\wedge$, respectively. A lattice is complete if every subset of the lattice has a unique supremum and infimum. This also implies that the entire lattice has a supremum and infimum, which are called the universal bounds of the lattice.

A lattice operator maps a lattice element into another lattice element. [Strictly speaking, a lattice operator maps an element of one (source) lattice to an element of another (target) lattice. The target lattice may be identical to or different from the source lattice. Here, we restrict ourselves to identical source and target lattices. This means that a lattice operation on a binary image will yield a binary image.] A dilation $\delta$ is a lattice operator that distributes over supremum and an erosion $\varepsilon$ is a lattice operator that distributes over infimum:

$\delta\left(\vee X_{i}\right)=\vee\left\{\delta\left(X_{i}\right)\right\}$

$\varepsilon\left(\wedge X_{i}\right)=\wedge\left\{\varepsilon\left(X_{i}\right)\right\}$

Some examples can clarify the concept of a lattice. The set of integers (augmented by $-\infty$ and $+\infty$ ) is a complete lattice because it does have structure: Any subset of integers can be ordered. The supremum and infimum operators correspond to the maximum and minimum operators. (The augmentation with $-\infty$ and $+\infty$ is necessary to provide the universal bounds of the lattice.) This is an example of a totally ordered lattice (also called a chain) in which the ordering relation applies to every pair of elements. Now consider the powerset of all $\mathbf{n} \times \mathbf{m}$ binary images; set inclusion is a relationship between members of this set that provides structure and ordering. A binary image $A$ is below $B(A<B)$ if $A \subset B$. This lattice is partially ordered (but not totally ordered) because there exists sets $A$ and $B$ for which the ordering relation does not hold; i.e., $A \nsubseteq B$ and $B \nsubseteq A$. For the binary image lattice, the supremum operator is set union and the infimum operator is set intersection: $A \vee B$ $=A \cup B$ and $A \wedge B=A \cap B$. The universal bounds of the binary image lattice are the empty set and the whole set.

Consider the binary image operations of Minkowski set addition and Minkowski set subtraction. These are restated, respectively, as

$$
X \oplus B=\underset{b \in B}{\cup} X_{b},
$$


$X \ominus B=\bigcap_{b \in B} X_{-b}$

It has been shown that Minkowski set addition distributes over set union and Minkowski set subtraction distributes over set intersection. Therefore, they are dilation and erosion operators for the binary image lattice.

In grayscale images, it is the ordering of the graylevels that provides the structure where one image $f$ is below another image $g(f<g)$ if $f(x) \leqslant g(x)$ for all $x$. The supremum and infimum operators are the pointwise maximum and minimum operators:

$f \vee g=\operatorname{maximum}\{f(x), g(x)\} \quad \forall x$

and

$f \wedge g=\operatorname{minimum}\{f(x), g(x)\} \forall x$.

Grayscale dilation and erosion are defined as

$$
\begin{aligned}
& {[f \oplus g](x)=\max _{\substack{z \in D \\
z-x \in G}}\{f(z)+g(z-x)\},} \\
& {[f \ominus g](x)=\min _{\substack{z \in D \\
z-x \in G}}\{f(z)-g(z-x)\},}
\end{aligned}
$$

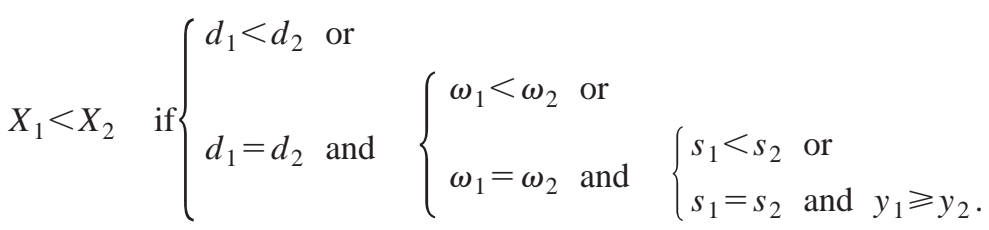

where $g(x)$ is a structuring function, $D$ is the image domain, and $G$ is the structuring element support. As for the binary case, it can be shown that dilation and erosion distribute, respectively, across supremum (maximum) and infimum (minimum) operations.

It is important to recognize that it is the set of all possible images (either binary or grayscale) that forms a partially ordered complete lattice. Morphological operators rely on the total ordering of the pixel values: the binary set $\{0,1\}$ or the scalar intensity range.

The proposed color morphological operators must also fit within the lattice theory framework for general morphology. The lattice framework does not preclude the possibility of generating an output value (via the supremum or infimum) that is not present in the input. However, to avoid the introduction of color artifacts, especially at the boundary between objects of different colors, we have constrained the output of color morphological operations to be one of the input values. Imposing this constraint required that the color vectors be totally ordered, which we have done using conditional ordering of $(d, \omega, s, y)$. To prove that the set of color vectors is totally ordered, we must show that the properties of reflexivity, antisymmetry, transitivity, and pairwise ordering hold. The proposed ordering relation for color vectors is restated below.
If $X_{2}$ and $\left(d_{2}, \omega_{2}, s_{2}, y_{2}\right)$ are replaced by $X_{1}$ and $\left(d_{1}, \omega_{1}, s_{1}, y_{1}\right)$ in the ordering relation, then $X_{1}<X_{1}$ because $d_{1}=d_{1}, \omega_{1}=\omega_{1}, s_{1}=s_{1}$, and $y_{1}=y_{1}$. Therefore, the ordering relation is reflexive.

To show that the ordering relation is antisymmetric, we assume that $X_{1}<X_{2}$ and $X_{2}<X_{1}$. Both assumptions can be true only if $d_{1}=d_{2}, \omega_{1}=\omega_{2}, s_{1}=s_{2}$, and $y_{1}=y_{2}$, and therefore $X_{1}=X_{2}$ and the ordering relation is antisymmetric.

To show that the ordering relation is transitive, assume that $X_{1}<X_{2}$ and $X_{2}<X_{3}$. Furthermore, assume that $d_{1}$ $<d_{2}$ and $d_{2}<d_{3}$. By transitivity of real numbers, $d_{1}<d_{3}$ and therefore $X_{1}<X_{3}$. This same argument extends to $\omega, s$, and $y$ in the case of equality in the lower-precedence components. Therefore, the ordering relation is transitive.

Because all color vectors have corresponding $(d, \omega, s, y)$ real-valued measurements, the ordering relation applies to any pair of color vectors $X_{1}$ and $X_{2}$; i.e., $X_{1}<X_{2}$ or $X_{2}$ $<X_{1}$. Because the ordering relation is reflexive, antisymmetric, transitive, and pairwise ordered for the set of color vectors, then the set of color vectors is a totally ordered set.

Because of the total ordering of color vectors, color images can be partially ordered. The set of color images then forms a complete lattice, just as the set of grayscale images forms a complete lattice because of the total ordering of the graylevels. Color image $\mathbf{f}$ is below color image $\mathbf{g}$ if $\mathbf{f}(x)$ $<\mathbf{g}(x) \forall x$. The supremum and infimum operators for the color image lattice are obtained from pointwise supremum and infimum operators:

$(\mathbf{f} \vee \mathbf{g})(x)=\mathbf{f}(x) \vee \mathbf{g}(x)$

$(\mathbf{f} \wedge \mathbf{g})(x)=\mathbf{f}(x) \wedge \mathbf{g}(x)$.

Having shown that the set of color images forms a lattice using the proposed ordering of color vectors, it remains to be seen that the proposed dilation and erosion operators also fit within the lattice framework. We can show that the dilation operator for color images distributes over supremum as required by lattice theory:

$\delta\left(\vee X_{i}\right)=\vee\left\{\delta\left(X_{i}\right)\right\}$

Let $X_{i}, i=1 \ldots M$, be a set of $M$ color images. The supremum of set $X_{i}, \vee X_{i}$, is a color image created by taking the pointwise supremum: 


$$
\begin{aligned}
\left(\vee X_{i}\right)(x, y) & =X_{1}(x, y) \vee X_{2}(x, y) \vee \ldots \vee X_{M}(x, y) \\
& =X_{j}(x, y): d_{j}(x, y)<d_{i}(x, y) \quad \forall i \neq j .
\end{aligned}
$$

That is, the output is chosen by conditionally ordering the color vectors $X_{i}(x, y)$ from all images $i$ at each pixel location $(x, y)$ according to $(\min d, \min \omega, \min s, \max y)$ and selecting the color pixel corresponding to the first order statistic. (In the case of equal distances, the successive components are used for ordering; this is not shown in the above equation to keep the proof concise.) Let $W[x, y]$ be the set of coordinates in the structuring element support around pixel location $(x, y)$ (i.e., $W$ is the operator's window). The dilation of the resulting image $\delta\left(\vee X_{i}\right)$ conditionally orders all color vectors within the window $W[x, y]$ of each pixel location $x, y$ according to $(\min d, \min \omega, \min$ $s$, max $y$ ) and selects the color pixel corresponding to the first order statistic

$$
\delta\left(\vee X_{i}\right)(x, y)=\underset{(w, z) \in W[x, y]}{\vee}\left(\vee X_{i}\right)(w, z) .
$$

The order of supremum operations can be transposed, and then the inner supremum operator is equivalent to dilation of each image $X_{i}$

$$
\begin{aligned}
& \delta\left(\vee X_{i}\right)(x, y)=\underset{(w, z) \in W[x, y]}{\vee}\left(\underset{i}{\left.\left(\vee X_{i}\right)(w, z)\right)}\right. \\
& =\vee\left(\underset{i}{\vee} \underset{(w, z) \in W[x, y]}{\vee} X_{i}(w, z)\right)=\underset{i}{\vee}\left(\delta\left(X_{i}\right)\right) .
\end{aligned}
$$

Both operations $\delta\left(\vee X_{i}\right)$ and $\vee\left[\delta\left(X_{i}\right)\right]$ reduce to selection of the first order statistic from the conditional ordering of the set $\cup_{i} \cup_{(w, z) \in W[x, y]}\left(X_{i}(w, z)\right)$ of color vectors. In a similar fashion, it can be shown that the erosion operator distributes over infimum.

\section{References}

1. A. R. Weeks, Fundamentals of Electronic Image Processing, pp. 109-120, SPIE, Piscataway, NJ (1996).

2. H. Heijmans, Morphological Image Operators, Academic, New York, (1994).
3. J. Serra, Anamorphoses and Function Lattices (Multivalued Morphology), Mathematical Morphology in Image Processing, E. Dougherty, Ed., pp. 483-521, (1993).

4. P. E. Trahanias and A. N. Venetsanopoulos, "Color edge detectors based on multivariate ordering,' Visual Communications and Image Processing, Vol. 1818, Dekker, New York (1992).

5. V. Barnett, "The ordering of multivariate data,' J. R. Stat. Soc. A. 139(3), 318-343 (1976).

6. I. Pitas, "Marginal order statistics in color image filtering,', Opt. Eng. 29, 495-503 (1990).

7. I. Pitas and P. Tsakalides, "Multivariate ordering in color image restoration,' in Proc. IEEE Transactions on Circuits and Systems for Video Technology, pp. 247-260 (Sept. 1991).

8. J. Astola, P. Haavisto, and Y. Neuvo, "Vector median filters" Proc. IEEE 78, 678-689 (1990).

9. P. E. Trahanias and A. N. Venetsanopoulos, "Multispectral image processing,' Image Processing: Theory and Applications, Elsevier Science, New York (1993)

10. M. L. Comer and E. J. Delp, "Morphological operations for color image processing,' J. Electron. Imaging 8, 279-289 (1999).

11. R. A. Peters II, in Mathematical Morphology for Angle-Valued Images, in Proc. SPIE 3026, 84-94 (1997).

12. A. V. J. Martin, Technical Television, pp. 498-503, Prentice-Hall, Englewood Cliffs, NJ (1962).

13. P. Maragos, "Pattern spectrum and multiscale shape representation," IEEE Trans. Pattern Anal. Mach. Intell. 11(7), 701-716 (1989).

14. H. de Ridder, F. J. J. Blommaert, and E. A. Fedorovskaya, "Naturalness and image quality: Chroma and hue variation in color images of natural scenes,' Proc. SPIE 2411, 51-61 (1994).

15. D. E. Rutherford, Introduction to Lattice Theory, Oliver and Boyd, London (1965).

Lloyd J. Sartor received his PhD in electrical engineering from the University of Central Florida in 2000 and his BSEE and MSEE degrees from Southern Methodist University in 1986 and 1988, respectively. He is employed at Siemens Information and Communication Networks working on voice-over-packet technology. His current research interests are color image processing and morphological image processing.

Arthur R. Weeks received his $\mathrm{PhD}$ in electrical engineering from the University of Central Florida in Orlando in 1987. After completion of his PhD, he spent one year at the Royal Signals and Radar Establishment in Malvern, England studying laser beam propagation. $\mathrm{He}$ is presently an associate professor of electrical and computer engineering at the University of Central Florida. Dr. Weeks' interest include color image processing techniques, the reduction of noise within images using adaptive nonlinear filters, and the use of artificial neural networks in pattern recognition. Dr. Weeks is a member of the IEEE, SPIE, and Tau Beta Pi. 